\title{
Time-reversal symmetry breaking versus chiral symmetry breaking in twisted bilayer graphene
}

\author{
J. González $\oplus^{1}$ and T. Stauber ${ }^{2}$ \\ ${ }^{1}$ Instituto de Estructura de la Materia, CSIC, E-28006 Madrid, Spain \\ ${ }^{2}$ Materials Science Factory, Instituto de Ciencia de Materiales de Madrid, CSIC, E-28049 Madrid, Spain
}

(Received 5 June 2020; revised 7 August 2020; accepted 11 August 2020; published 25 August 2020)

\begin{abstract}
By applying a self-consistent Hartree-Fock approximation, we show that the mechanism of dynamical symmetry breaking can account for the insulating phase that develops about the charge neutrality point of twisted bilayer graphene around the magic angle. (i) If the Coulomb interaction is screened by metallic gates, the opening of a gap between the lowest-energy valence and conduction bands proceeds through the breakdown of chiral symmetry at strong coupling. Increasing the dielectric screening, however, we find a critical coupling at which chiral symmetry breaking is suppressed, triggering a very strong signal for time-reversal symmetry breaking with Haldane mass. (ii) If the long-range tail of the Coulomb interaction is not screened, we see the appearance of yet a different dominant pattern at strong coupling, which is characterized by breaking the time-reversal invariance but with opposite flux in the two sublattices of each carbon layer, with the consequent valley symmetry breaking. In this case a gap is also opened in the Dirac cones, but superposed to the splitting of the degeneracy of the low-energy bands at the $K$ points of the moiré Brillouin zone.
\end{abstract}

DOI: 10.1103/PhysRevB.102.081118

Introduction. The discovery of superconductivity [1] next to insulating phases [2] in twisted bilayer graphene (TBG) at small twist angles has opened a new era in the investigation of strong electron correlations in two-dimensional (2D) materials [3-34]. The strong correlation effects appear at integer fillings of the superlattice of TBG, suggesting that the large effective strength of the Coulomb interaction is the driving force behind them. Furthermore, band renormalization has recently been verified in local probe experiments [35-38].

A remarkable effect is the insulating behavior observed at the charge neutrality point (CNP) of TBG [7], when the Fermi level is placed in the undoped carbon material at the vertices of the Dirac cones characterizing the low-energy dispersion in the moiré Brillouin zone (MBZ). As long as the density of states vanishes at that filling level, it is pertinent to ask about the mechanism responsible for the opening of a gap at the CNP.

This discussion recalls the highly debated question about the dynamical breakdown of chiral symmetry in Dirac-like systems [39-46]. That kind of electronic instability has not been observed in graphene, despite the large nominal coupling of the Coulomb potential in the carbon layer [47-49] (nevertheless, correlated insulated states have been seen in bilayer graphene [50,51]). In the case of TBG, the relative strength of the Coulomb interaction is further enhanced at the magic angle, making plausible that the critical coupling for the opening of a gap at the Dirac cones may be surpassed.

In this Rapid Communication, we study the effects of the Coulomb interaction at the CNP of TBG near the magic angle, with the aim of discerning whether dynamical symmetry breaking takes place under different screening conditions. We adopt a tight-binding approach to make a real-space description of the system, and we resort to a Hartree-Fock approximation in order to assess the effects of the Coulomb interaction [36,38,52-55]. This approach allows us to treat the on-site Hubbard and long-range Coulomb interaction on the same footing.

TBG is a complex system where we find the interplay between different degrees of freedom (two valleys, two layers), leading to a number of condensates signaling the breakdown of symmetry. The resulting picture is that several transitions take place between different phases as one modifies the strength of the Coulomb interaction. This mainly favors the breakdown of chiral and time-reversal symmetry, whose order parameters tend to compete along the phase diagram and become alternatively dominant at different regimes of the coupling strength.

Hartree-Fock approximation. We focus our analysis on a twisted bilayer corresponding to $i=28$ (twist angle $\left.\theta \approx 1.16^{\circ}\right)$ in the sequence of commensurate superlattices with twist angle $\theta_{i}=\arccos \left[\left(3 i^{2}+3 i+0.5\right) /\left(3 i^{2}+3 i+1\right)\right]$ [56-58]. Our starting point to model this bilayer is a tightbinding approach, as described in detail in the Supplemental Material (SM) [59]. The Hamiltonian $H_{0}$ of the noninteracting theory is written in terms of creation (annihilation) operators $a_{i \sigma}^{\dagger}\left(a_{i \sigma}\right)$ for electrons at each site $i$ with spin $\sigma$ as $[60,61]$

$$
\begin{aligned}
H_{0}= & -\sum_{\langle i, j\rangle} t_{\|}\left(\boldsymbol{r}_{i}-\boldsymbol{r}_{j}\right)\left(a_{i \sigma}^{\dagger} a_{j \sigma}+\text { H.c. }\right) \\
& -\sum_{(i, j)} t_{\perp}\left(\boldsymbol{r}_{i}-\boldsymbol{r}_{j}\right)\left(a_{i \sigma}^{\dagger} a_{j \sigma}+\text { H.c. }\right),
\end{aligned}
$$

where the sum over the brackets $\langle\cdots\rangle$ runs over pairs of atoms in the same layer, whereas the sum over the curved brackets $(\cdots)$ runs over pairs with atoms belonging to different layers. $t_{\|}(\boldsymbol{r})$ and $t_{\perp}(\boldsymbol{r})$ are hopping matrix elements which have an 
exponential decay with the distance $|\boldsymbol{r}|$ between carbon atoms $[62,63]$. Our tight-binding model of TBG leads then to a combined bandwidth for the first valence and conduction bands adding up to a few meV (see the SM [59]).

On top of that noninteracting model, we incorporate the effect of the Coulomb potential $v(\boldsymbol{r})$. Then we add to $H_{0}$ the interaction Hamiltonian

$$
H_{\mathrm{int}}=\frac{1}{2} \sum_{i, j} a_{i \sigma}^{\dagger} a_{i \sigma} v\left(\boldsymbol{r}_{i}-\boldsymbol{r}_{j}\right) a_{j \sigma^{\prime}}^{\dagger} a_{j \sigma^{\prime}} .
$$

We face the many-body problem with the aim of computing the electron propagator $G$ of the interacting theory. This can be obtained in terms of the propagator $G_{0}$ of the noninteracting system and the electron self-energy $\Sigma$ according to the Dyson equation

$$
G^{-1}=G_{0}^{-1}-\Sigma .
$$

In what follows we are going to be interested in timeindependent observables, so that we can simplify the discussion by taking the zero-frequency (static) limit of the equations. In that limit, the free propagator $G_{0}$ is given in real space by the inverse of the matrix representation of the operator $H_{0}$, that is,

$$
\left(G_{0}\right)_{i j}=-\left(H_{0}^{-1}\right)_{i j}
$$

In terms of the eigenvalues $\varepsilon_{a}^{0}$ and eigenvectors $\phi_{a}^{0}\left(\boldsymbol{r}_{i}\right)$ of the noninteracting Hamiltonian, we have therefore in the static limit

$$
\left(G_{0}\right)_{i j}=-\sum_{a} \frac{1}{\varepsilon_{a}^{0}} \phi_{a}^{0}\left(\boldsymbol{r}_{i}\right) \phi_{a}^{0}\left(\boldsymbol{r}_{j}\right)^{*} .
$$

The Hartree-Fock approximation amounts to assuming that the full propagator can be represented in terms of a set of modified eigenvalues $\varepsilon_{a}$ and eigenvectors $\phi_{a}\left(\boldsymbol{r}_{i}\right)$ :

$$
(G)_{i j}=-\sum_{a} \frac{1}{\varepsilon_{a}} \phi_{a}\left(\boldsymbol{r}_{i}\right) \phi_{a}\left(\boldsymbol{r}_{j}\right)^{*} .
$$

One can check (in the full time-dependent theory) that the assumption in Eq. (6) implies the representation of the selfenergy matrix [64]

$$
\begin{aligned}
\Sigma_{i j}= & 2 \mathbb{I}_{i j} \sum_{l} v\left(\boldsymbol{r}_{i}-\boldsymbol{r}_{l}\right) \sum_{a}^{\prime}\left|\phi_{a}\left(\boldsymbol{r}_{l}\right)\right|^{2} \\
& -v\left(\boldsymbol{r}_{i}-\boldsymbol{r}_{j}\right) \sum_{a}^{\prime} \phi_{a}\left(\boldsymbol{r}_{i}\right) \phi_{a}\left(\boldsymbol{r}_{j}\right)^{*},
\end{aligned}
$$

where the prime means that the sum is to be carried over the occupied levels. The problem reverts to the fact that (3) and (7) become now a set of self-consistent equations. In practice, one can devise a recursive approximation to $G$ in which good convergence is achieved by building the self-energy at each step with the eigenvectors obtained in the previous iteration.

Orderparameters. In Fig. 1, we schematically show the relevant order parameters as they arise in single-layer graphene, namely, the chiral symmetry breaking giving rise to a Dirac mass [65] and the time-reversal symmetry breaking giving rise to the Haldane mass [66]. We will now extend this analysis to TBG within the Hartree-Fock approximation, in which the (a)

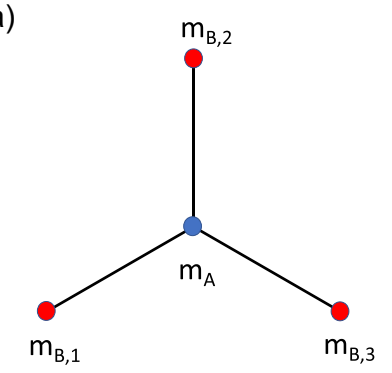

$m_{\text {chiral }}=m_{A}-\left(m_{B, 1}+m_{B, 2}+m_{B, 3}\right) / 3$ (b)

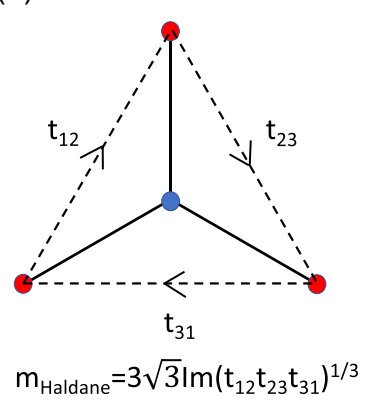

FIG. 1. Schematic definition of the two main symmetry-breaking patterns in the honeycomb lattice: (a) chiral symmetry breaking leading to a Dirac mass (where we have replaced $h_{i i}$ defined in the main text by $m$ ) and (b) time-reversal symmetry breaking leading to the Haldane mass (where we have replaced $h_{i j}$ defined in the main text by $t_{i j}$ ). Note that the factor $3 \sqrt{3}$ coming from the Haldane model is neglected in our general definition of Eqs. (10) and (11).

different symmetry-breaking patterns can be cast in terms of the matrix elements

$$
h_{i j}=\sum_{a}^{\prime} \phi_{a}\left(\boldsymbol{r}_{i}\right) \phi_{a}\left(\boldsymbol{r}_{j}\right)^{*},
$$

where the prime means again that the sum is only over occupied states. We note that we have actually the correspondence $h_{i j}=\left\langle a_{j}^{\dagger} a_{i}\right\rangle$, as shown in the SM [59].

In the present case, we have sublattices $A_{1}, B_{1}$ for the top carbon layer and $A_{2}, B_{2}$ for the bottom layer. Thus, we may write two different order parameters for the breakdown of chiral symmetry, being respectively even and odd under the exchange of the two layers, as

$$
C_{ \pm}=\sum_{i \in A_{1}} h_{i i}-\sum_{i \in B_{1}} h_{i i} \pm\left(\sum_{i \in A_{2}} h_{i i}-\sum_{i \in B_{2}} h_{i i}\right) .
$$

Furthermore, we have two different order parameters for the breakdown of time-reversal invariance (and parity), being respectively even and odd under the exchange of the two layers. We may label, for instance, the three nearest neighbors of each atom $i$ of the twisted bilayer as $i_{1}, i_{2}$ and $i_{3}$, with the order of the labels corresponding to clockwise orientation. We then have the two choices

$$
\begin{aligned}
P_{ \pm}= & \operatorname{Im}\left[\sum_{i \in A_{1}}\left(h_{i_{1} i_{2}} h_{i_{2} i_{3}} h_{i_{3} i_{1}}\right)^{1 / 3}+\sum_{i \in B_{1}}\left(h_{i_{1} i_{2}} h_{i_{2} i_{3}} h_{i_{3} i_{1}}\right)^{1 / 3}\right. \\
& \left. \pm \sum_{i \in A_{2}}\left(h_{i_{1} i_{2}} h_{i_{2} i_{3}} h_{i_{3} i_{1}}\right)^{1 / 3} \pm \sum_{i \in B_{2}}\left(h_{i_{1} i_{2}} h_{i_{2} i_{3}} h_{i_{3} i_{1}}\right)^{1 / 3}\right] .
\end{aligned}
$$

The imaginary part of the terms in Eq. (10) gives a measure of the flux through the twisted bilayer, providing a signature of the breakdown of time-reversal invariance. However, we may also envisage the possibility that the direction of the flux may be the opposite in sublattices $A$ and $B$. That pattern corresponds to a different type of breakdown of time-reversal symmetry. The order parameter for that phase may be even or odd with respect to the exchange of the two carbon layers, 
which translates into the two possibilities

$$
\begin{aligned}
S_{ \pm}= & \operatorname{Im}\left[\sum_{i \in A_{1}}\left(h_{i_{1} i_{2}} h_{i_{2} i_{3}} h_{i_{3} i_{1}}\right)^{1 / 3}-\sum_{i \in B_{1}}\left(h_{i_{1} i_{2}} h_{i_{2} i_{3}} h_{i_{3} i_{1}}\right)^{1 / 3}\right. \\
& \left. \pm \sum_{i \in A_{2}}\left(h_{i_{1} i_{2}} h_{i_{2} i_{3}} h_{i_{3} i_{1}}\right)^{1 / 3} \mp \sum_{i \in B_{2}}\left(h_{i_{1} i_{2}} h_{i_{2} i_{3}} h_{i_{3} i_{1}}\right)^{1 / 3}\right] .
\end{aligned}
$$

The development of nonvanishing order parameters $S_{ \pm}$ leads to the splitting of the low-energy bands at the $K$ points of the MBZ. This is due to the fact that, in the low-energy theory of Dirac fermions, such a breakdown of symmetry proceeds with the dynamical generation of a term proportional to the identity in pseudospin space. This term preserves the Dirac nodes at the $K$ points, but leading to a different shift of the bands in the two valleys of the twisted bilayer, thus effectively breaking valley symmetry.

Besides, we have to include the possibility of having a spontaneous imbalance of charge in the two carbon layers as a result of interaction effects. The order parameter for such a symmetry breaking is

$$
L=\sum_{i \in A_{1}} h_{i i}+\sum_{i \in B_{1}} h_{i i}-\sum_{i \in A_{2}} h_{i i}-\sum_{i \in B_{2}} h_{i i} .
$$

This completes the list of order parameters described from a real-space point of view and which preserve the threefold rotational symmetry. We capture in this way the symmetrybreaking patterns which have a most drastic effect on the low-energy bands, with the potential to destabilize the Dirac nodes at the CNP [67].

Screened Coulomb interaction. We first consider a form of the Coulomb potential which is adapted to the case where TBG is surrounded by top and bottom metallic gates. The case of a setup with a single metallic gate leads to results similar to those presented here, as shown in the SM [59]. We start with the unscreened Coulomb potential $v_{0}(\boldsymbol{r})=e^{2} / 4 \pi \epsilon r, \epsilon$ being the dielectric constant of the surrounding (nonmetallic) medium. Taking into account the presence of the two gates, each at a distance $d=\xi / 2$ from the twisted bilayer, it can be shown that the electrostatic potential becomes [68]

$$
v(\boldsymbol{r})=\frac{e^{2}}{4 \pi \epsilon} \frac{2 \sqrt{2} e^{-\pi r / \xi}}{\xi \sqrt{r / \xi}} .
$$

In this section, we consider in particular a setup with $\xi=10$ nm.

Furthermore, the potential has to be still complemented with the value of the Coulomb repulsion for electrons at the same carbon atom, as the expression (13) is ill-defined for $\boldsymbol{r}=0$. We have taken such an on-site Coulomb repulsion equal to $8 \mathrm{eV}$, i.e., we have regularized the Coulomb potential with the prescription $\left.v(r)\right|_{r=0}=8 \mathrm{eV}$, irrespective of the value of $\epsilon$.

We have mapped the different symmetry-breaking patterns as the strength of the Coulomb potential is modified, which may be achieved in practice by changing the dielectric constant $\epsilon$. The evolution of the relevant order parameters can be seen in Fig. 2(a). There, we observe that the order parameter for the breakdown of chiral symmetry, $C_{+}$, becomes (a)

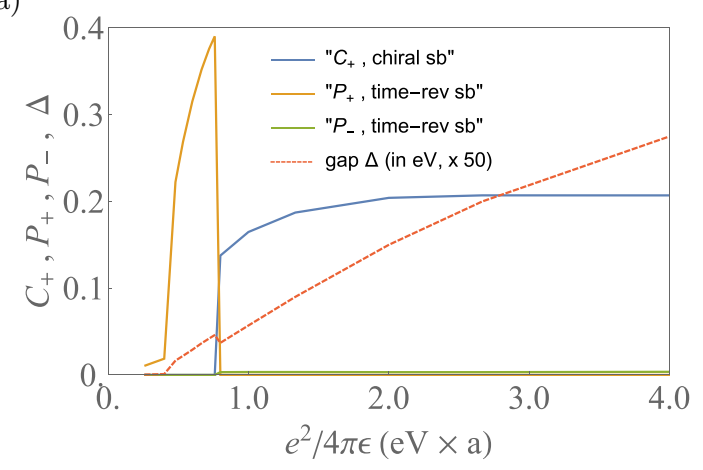

(b)

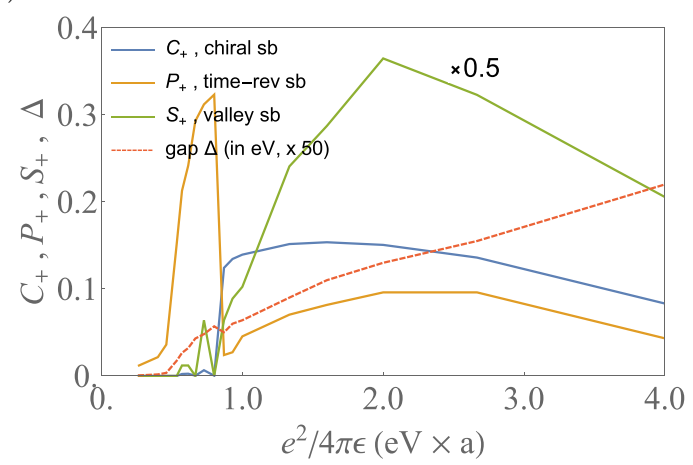

FIG. 2. Plot of the order parameters characterizing the dominant symmetry-breaking patterns of twisted bilayer graphene with $i=28$ at the charge neutrality point as a function of the coupling of the Coulomb potential, i.e., the dielectric constant $\epsilon$ (in units where $a$ is the C-C distance): (a) Coulomb potential screened by metallic gates and (b) unscreened long-ranged Coulomb potential. For a boron nitride dielectric, we have $e^{2} / 4 \pi \epsilon \approx 2 \mathrm{eV} \times a$, predicting a clear difference between the screened and unscreened setups.

dominant for strong and intermediate coupling of the Coulomb potential.

However, we see also the development of a strong peak of $P_{+}$at large $\epsilon$, right at the point where the order parameter $C_{+}$is suppressed. This reflects the competition and even mutual exclusion between time-reversal and chiral symmetry breaking in the model with the screened Coulomb interaction. We stress that the peak of $P_{+}$is not significantly altered if the on-site (unscreened) repulsion $v(0)$ is switched off, which means that the strong peak is due to the extended component of the Coulomb interaction.

Concomitant with the two mentioned symmetry-breaking patterns, we find the development of a gap at the $K$ point of the MBZ, plotted also in Fig. 2(a). The magnitude of the gap for a given coupling strength remains even in almost the whole MBZ, as can be seen in the band dispersion shown in Fig. 3(a). The gap only closes at a single point (the $\Gamma$ point) in the discretization we have used for the MBZ, from which we infer that the results could still be compatible with the insulating behavior at the CNP upon extrapolation to larger grids.

Long-range Coulomb interaction. It is also interesting to study the breakdown of symmetry when TBG is not surrounded by metallic gates. In this case the Coulomb potential 
(a)

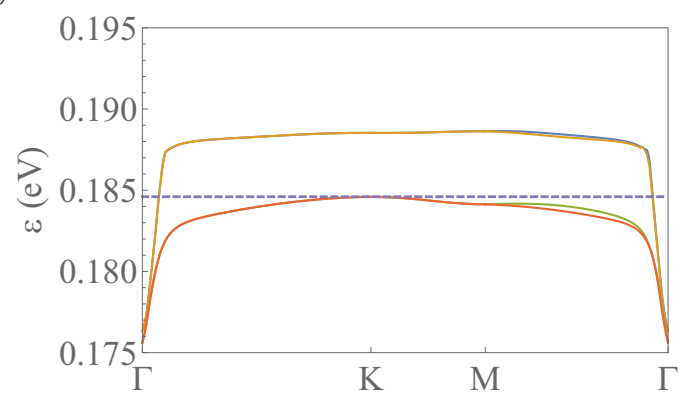

(b)

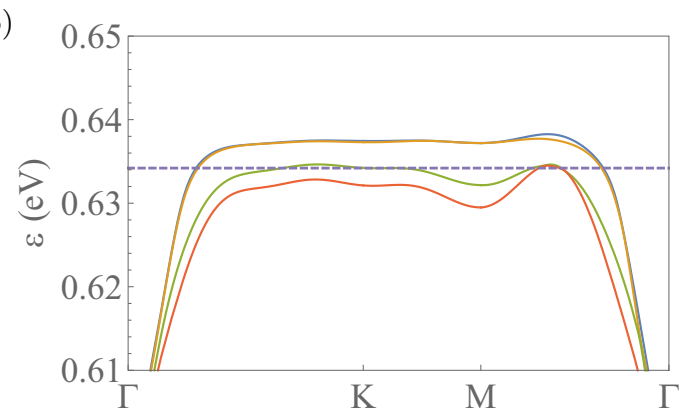

FIG. 3. Dispersion of the first valence and conduction bands about the charge neutrality point of a twisted bilayer with $\theta_{28} \approx$ $1.16^{\circ}$, computed in a Hartree-Fock approximation with (a) the screened potential in Eq. (13) and (b) the unscreened long-range potential in (14), for the same coupling strength $e^{2} / 4 \pi \epsilon \approx 2.66 \mathrm{eV} \times a$ (corresponding to a $\mathrm{Si}$ substrate). The dashed line stands for the Fermi level, placed at the energy of the highest valence band at the $K$ point.

is given by

$$
v(\boldsymbol{r})=\frac{e^{2}}{4 \pi \epsilon} \frac{1}{r},
$$

with the screening effects just encoded in the dielectric constant $\epsilon$. As in the previous section, we also assume that the interaction at $\boldsymbol{r}=0$ is regularized to coincide with the on-site Coulomb repulsion of electrons at the same carbon atom, which we take as $8 \mathrm{eV}$.

The competition between the relevant symmetry-breaking patterns can be seen in Fig. 2(b), in which we have plotted the different behaviors as the coupling of the Coulomb potential is modified by $\epsilon$. The most remarkable feature observed is the prevalence of the pattern that breaks time-reversal symmetry but with opposite flux in the two sublattices of each carbon layer, characterized by the order parameter $S_{+}$, which is the dominant order parameter in the strong-coupling regime.
As mentioned before, a nonvanishing value of $S_{+}$does not contribute to opening a gap in the Dirac cones at the $K$ points, although it leads to valley symmetry breaking. This means that the gap seen at strong coupling in Fig. 2(b) must come from the coexistent chiral and time-reversal symmetry breaking characterized by $C_{+}$and $P_{+}$. The dispersion of the low-energy bands, represented in a typical strong-coupling situation in Fig. 3(b), shows indeed the development of a gap in a large portion of the MBZ, together with the splitting of the valence bands driven by the symmetry-breaking pattern $S_{+}$[69]. Comparing with the bands in Fig. 3(a), we observe that the magnitude of the gap about the $K$ point is very similar in the two cases, while the bands are pushed higher in Fig. 3(b) as a consequence of the higher electrostatic energy from the long-range tail of the interaction.

Summary. We have shown that the mechanism of dynamical symmetry breaking can account for the opening of a gap in the Dirac cones at the CNP of TBG near the magic angle. Our study also stresses the relevance of the experimental conditions by which the Coulomb interaction is screened. When TBG is surrounded by nearby metallic gates, we find a transition from time-reversal to chiral symmetry breaking at intermediate coupling. But in a more ideal case where the long-range tail of the Coulomb interaction is not screened, we see the prevalence of a different pattern at strong coupling, characterized by breaking the time-reversal invariance but with opposite flux in the two sublattices of each carbon layer, with the consequent valley symmetry breaking.

The unscreened long-range interaction gives rise then to the superposition of two different effects at strong coupling, namely, the opening of a gap in the Dirac cones and the splitting of the degeneracy of the low-energy bands at the $K$ points. It also leads to a strong downward renormalization of the lowest conduction bands in their way towards the $\Gamma$ point, forcing them to cross the Fermi level. This feature in particular suggests that the symmetry breaking driven by the unscreened long-range Coulomb interaction would not be consistent with insulating behavior at the CNP of TBG.

It would be interesting to investigate similar symmetrybreaking effects at other filling levels of TBG. This could clarify whether other strongly correlated phenomena may bear some connection with dynamical symmetry breaking, and whether this effect may be enhanced at filling factors leading to a large density of states like that reached at the van Hove singularities in the low-energy bands.

Acknowledgments. This work has been supported by Spain's MINECO under Grant No. FIS2017-82260-P as well as by the CSIC Research Platform on Quantum Technologies PTI-001. Access to the computational resources of CESGA (Centro de Supercomputación de Galicia) is also gratefully acknowledged.
[1] Y. Cao, V. Fatemi, S. Fang, K. Watanabe, T. Taniguchi, E. Kaxiras, and P. Jarillo-Herrero, Nature (London) 556, 43 (2018).

[2] Y. Cao, V. Fatemi, A. Demir, S. Fang, S. L. Tomarken, J. Y. Luo, J. D. Sanchez-Yamagishi, K. Watanabe, T. Taniguchi,
E. Kaxiras, R. C. Ashoori, and P. Jarillo-Herrero, Nature (London) 556, 80 (2018).

[3] M. Yankowitz, S. Chen, H. Polshyn, Y. Zhang, K. Watanabe, T. Taniguchi, D. Graf, A. F. Young, and C. R. Dean, Science 363, 1059 (2019). 
[4] S. Moriyama, Y. Morita, K. Komatsu, K. Endo, T. Iwasaki, S. Nakaharai, Y. Noguchi, Y. Wakayama, E. Watanabe, D. Tsuya, K. Watanabe, and T. Taniguchi, arXiv:1901.09356.

[5] E. Codecido, Q. Wang, R. Koester, S. Che, H. Tian, R. Lv, S. Tran, K. Watanabe, T. Taniguchi, F. Zhang, M. Bockrath, and C. N. Lau, Sci. Adv. 5, eaaw9770 (2019).

[6] C. Shen, Y. Chu, Q. Wu, N. Li, S. Wang, Y. Zhao, J. Tang, J. Liu, J. Tian, K. Watanabe, T. Taniguchi, R. Yang, Z. Y. Meng, D. Shi, O. V. Yazyev, and G. Zhang, Nat. Phys. 16, 520 (2020).

[7] X. Lu, P. Stepanov, W. Yang, M. Xie, M. A. Aamir, I. Das, C. Urgell, K. Watanabe, T. Taniguchi, G. Zhang, A. Bachtold, A. H. MacDonald, and D. K. Efetov, Nature (London) 574, 653 (2019).

[8] G. Chen, A. L. Sharpe, P. Gallagher, I. T. Rosen, E. J. Fox, L. Jiang, B. Lyu, H. Li, K. Watanabe, T. Taniguchi, J. Jung, Z. Shi, D. Goldhaber-Gordon, Y. Zhang, and F. Wang, Nature (London) 572, 215 (2019).

[9] C. Xu and L. Balents, Phys. Rev. Lett. 121, 087001 (2018).

[10] G. E. Volovik, JETP Lett. 107, 516 (2018).

[11] N. F. Q. Yuan and L. Fu, Phys. Rev. B 98, 045103 (2018).

[12] H. C. Po, L. Zou, T. Senthil, and A. Vishwanath, Phys. Rev. B 99, 195455 (2019).

[13] B. Roy and V. Juričić, Phys. Rev. B 99, 121407(R) (2019).

[14] H. Guo, X. Zhu, S. Feng, and R. T. Scalettar, Phys. Rev. B 97, 235453 (2018).

[15] J. F. Dodaro, S. A. Kivelson, Y. Schattner, X. Q. Sun, and C. Wang, Phys. Rev. B 98, 075154 (2018).

[16] G. Baskaran, arXiv:1804.00627.

[17] C.-C. Liu, L.-D. Zhang, W.-Q. Chen, and F. Yang, Phys. Rev. Lett. 121, 217001 (2018).

[18] K. Slagle and Y. B. Kim, SciPost Phys. 6, 016 (2019).

[19] T. J. Peltonen, R. Ojajärvi, and T. T. Heikkilä, Phys. Rev. B 98, 220504(R) (2018).

[20] D. M. Kennes, J. Lischner, and C. Karrasch, Phys. Rev. B 98, 241407(R) (2018).

[21] M. Koshino, N. F. Q. Yuan, T. Koretsune, M. Ochi, K. Kuroki, and L. Fu, Phys. Rev. X 8, 031087 (2018).

[22] J. Kang and O. Vafek, Phys. Rev. X 8, 031088 (2018).

[23] H. Isobe, N. F. Q. Yuan, and L. Fu, Phys. Rev. X 8, 041041 (2018)

[24] F. Wu, A. H. MacDonald, and I. Martin, Phys. Rev. Lett. 121, 257001 (2018).

[25] Y.-H. Zhang, D. Mao, Y. Cao, P. Jarillo-Herrero, and T. Senthil, Phys. Rev. B 99, 075127 (2019).

[26] J. González and T. Stauber, Phys. Rev. Lett. 122, 026801 (2019).

[27] H. K. Pal, arXiv:1805.08803.

[28] M. Ochi, M. Koshino, and K. Kuroki, Phys. Rev. B 98, 081102(R) (2018).

[29] A. Thomson, S. Chatterjee, S. Sachdev, and M. S. Scheurer, Phys. Rev. B 98, 075109 (2018).

[30] S. Carr, S. Fang, P. Jarillo-Herrero, and E. Kaxiras, Phys. Rev. B 98, 085144 (2018).

[31] F. Guinea and N. R. Walet, Proc. Natl. Acad. Sci. USA 115, 13174 (2018).

[32] L. Zou, H. C. Po, A. Vishwanath, and T. Senthil, Phys. Rev. B 98, 085435 (2018).

[33] J. Kang and O. Vafek, Phys. Rev. Lett. 122, 246401 (2019).
[34] J. González and T. Stauber, Phys. Rev. Lett. 124, 186801 (2020).

[35] A. Kerelsky, L. J. McGilly, D. M. Kennes, L. Xian, M. Yankowitz, S. Chen, K. Watanabe, T. Taniguchi, J. Hone, C. Dean, A. Rubio, and A. N. Pasupathy, Nature (London) 572, 95 (2019).

[36] Y. Xie, B. Lian, B. Jäck, X. Liu, C.-L. Chiu, K. Watanabe, T. Taniguchi, B. A. Bernevig, and A. Yazdani, Nature (London) 572, 101 (2019).

[37] Y. Jiang, X. Lai, K. Watanabe, T. Taniguchi, K. Haule, J. Mao, and E. Y. Andrei, Nature (London) 573, 91 (2019).

[38] Y. Choi, J. Kemmer, Y. Peng, A. Thomson, H. Arora, R. Polski, Y. Zhang, H. Ren, J. Alicea, G. Refael, F. von Oppen, K. Watanabe, T. Taniguchi, and S. Nadj-Perge, Nat. Phys. 15, 1174 (2019).

[39] D. V. Khveshchenko, Phys. Rev. Lett. 87, 246802 (2001).

[40] T. Stauber, F. Guinea, and M. A. H. Vozmediano, Phys. Rev. B 71, 041406(R) (2005).

[41] V. P. Gusynin, V. A. Miransky, S. G. Sharapov, and I. A. Shovkovy, Phys. Rev. B 74, 195429 (2006).

[42] I. F. Herbut, Phys. Rev. B 75, 165411 (2007).

[43] J. E. Drut and T. A. Lähde, Phys. Rev. Lett. 102, 026802 (2009).

[44] O. V. Gamayun, E. V. Gorbar, and V. P. Gusynin, Phys. Rev. B 80, 165429 (2009).

[45] M. Trushin and J. Schliemann, Phys. Rev. Lett. 107, 156801 (2011).

[46] J. Jung and A. H. MacDonald, Phys. Rev. B 84, 085446 (2011).

[47] G. Li, A. Luican, and E. Y. Andrei, Phys. Rev. Lett. 102, 176804 (2009).

[48] D. A. Siegel, C.-H. Park, C. Hwang, J. Deslippe, A. V. Fedorov, S. G. Louie, and A. Lanzara, Proc. Natl. Acad. Sci. USA 108, 11365 (2011).

[49] J. Chae, S. Jung, A. F. Young, C. R. Dean, L. Wang, Y. Gao, K. Watanabe, T. Taniguchi, J. Hone, K. L. Shepard, P. Kim, N. B. Zhitenev, and J. A. Stroscio, Phys. Rev. Lett. 109, 116802 (2012).

[50] J. Martin, B. E. Feldman, R. T. Weitz, M. T. Allen, and A. Yacoby, Phys. Rev. Lett. 105, 256806 (2010).

[51] R. T. Weitz, M. T. Allen, B. E. Feldman, J. Martin, and A. Yacoby, Science 330, 812 (2010).

[52] T. Cea, N. R. Walet, and F. Guinea, Phys. Rev. B 100, 205113 (2019).

[53] L. Rademaker, D. A. Abanin, and P. Mellado, Phys. Rev. B 100, 205114 (2019).

[54] N. Bultinck, E. Khalaf, S. Liu, S. Chatterjee, A. Vishwanath, and M. P. Zaletel, Phys. Rev. X 10, 031034 (2020).

[55] M. J. Klug, New J. Phys. 22, 073016 (2020).

[56] J. M. B. Lopes dos Santos, N. M. R. Peres, and A. H. Castro Neto, Phys. Rev. Lett. 99, 256802 (2007).

[57] R. Bistritzer and A. H. MacDonald, Proc. Natl. Acad. Sci. USA 108, 12233 (2011).

[58] E. J. Mele, Phys. Rev. B 81, 161405(R) (2010).

[59] See Supplemental Material at http://link.aps.org/supplemental/ 10.1103/PhysRevB.102.081118 for more details and additional results.

[60] E. Suárez Morell, J. D. Correa, P. Vargas, M. Pacheco, and Z. Barticevic, Phys. Rev. B 82, 121407(R) (2010).

[61] G. T. de Laissardière, D. Mayou, and L. Magaud, Nano Lett. 10, 804 (2010). 
[62] I. Brihuega, P. Mallet, H. González-Herrero, G. Trambly de Laissardière, M. M. Ugeda, L. Magaud, J. M. GómezRodríguez, F. Ynduráin, and J.-Y. Veuillen, Phys. Rev. Lett. 109, 196802 (2012).

[63] P. Moon and M. Koshino, Phys. Rev. B 87, 205404 (2013).

[64] A. L. Fetter and J. D. Walecka, Quantum Theory of ManyParticle Systems (McGraw-Hill, New York, 1971).

[65] G. W. Semenoff, Phys. Rev. Lett. 53, 2449 (1984).

[66] F. D. M. Haldane, Phys. Rev. Lett. 61, 2015 (1988).
[67] We restrict here our analysis to those order parameters not involving the spin degree of freedom, which is justified as long as we deal with a dominant spin-independent interaction given by the Coulomb potential.

[68] R. E. Throckmorton and O. Vafek, Phys. Rev. B 86, 115447 (2012).

[69] A splitting of the low-energy bands is also observed in the density functional theory study reported by P. Lucignano, D. Alfè, V. Cataudella, D. Ninno, and G. Cantele, Phys. Rev. B 99, 195419 (2019). 